\title{
DESIGN OF AUTOMATIC CONTROL SYSTEM FOR INTELLIGENT CLEANING ROBOT OF CONDENSER
}

\author{
Tao Yan ${ }^{1 *}$, Lijun Sun ${ }^{1}$ \\ ${ }^{1}$ Yantai vocational college, Shandong, China \\ *Corresponding author: Tao Yan \\ Email: yantaowcw111@126.com
}

\begin{abstract}
In order to explore the design of automatic control system for intelligent cleaning robot of condenser, correlation analysis between fuzzy system and neural network system is made, and they are combined to design simulation model of control system model, and the learning algorithm and training conditions of the controller are analyzed. Finally, experiments verify that the fuzzy neural network controller can output the desired results. It is found that the output error of the fuzzy neural network is lower than the given error of 0.01 when the training error analysis of the fuzzy neural network is about 85 steps in the training process, so the correct and stable output of the fuzzy neural network can be obtained. When the step response analysis of the control system is carried out, the control system has reached a stable state when the time of the step response analysis is $1.6 \mathrm{~s}$, and the adjustment precision is high, the steady performance is good, and it has strong robustness. Therefore, the fuzzy algorithm is combined with the neural network to design the automatic control system of the intelligent cleaning robot for condenser, and the results obtained through the experimental simulation can output the desired results, so as to meet the requirements of precise automatic control positioning. Although there are still some shortcomings in the experimental process, it still provides a reference for the design of automatic control system of intelligent cleaning robot of condenser.
\end{abstract}

Keywords: Condenser; Neural Network; Intelligent Cleaning Robot; Automatic Control System.

\section{Introduction}

Today, with the development of society and the continuous progress of science and technology, intelligent robots are becoming more and more popular. Sweeping robots, palletizing robots, etc., are applied in more and more fields. Especially in some harsh environments, such as deep sea and high altitude, which are difficult for human beings to operate, the existence of robots is very important [1].

As one of the key equipment in thermal power, nuclear power, chemical industry and pharmaceutical industry, large condenser is mainly composed of tens of thousands of condensation tubes.

It plays the role of cooling source in the thermal cycle of steam turbine in thermal power plant. It also acts as cooling source in steam turbine, reduces the exhaust pressure and temperature of steam turbine, and improves the cycle thermal efficiency of generator set $[2,3]$.

During the operation of condenser, dirt accumulated on the inner wall of condensation tube is harmful to heat transfer due to unclean cooling water and chemical reaction during heat exchange [4].

The existence of fouling can cause a series of hazards, such as reducing the efficiency of steam turbine generator in thermal power plant, increasing the energy consumption of circulating water pump, causing condensation pipe blockage and even causing corrosion perforation of pipe wall, which can cause accidents [5]. The use of cleaning robots can not only save manpower, but also greatly improve work efficiency. For the cleaning of dirt, rubber ball cleaning or manual cleaning are mostly used at present [6].

There are some shortcomings in rubber ball cleaning, such as low recovery rate of rubber ball, easy blockage of condensation tube, inability to completely remove crystallization dirt caused by chemical reaction, and so on. So far, the ideal effect cannot be achieved: manual cleaning is difficult to meet the needs of large-scale production because of the large number of condensation tubes, long length, high labour intensity, poor working environment of workers, and needs unit shutdown or load reduction operation, which is difficult to meet the 
requirements of large-scale production and safety production requirements $[7,8]$.

At present, the application of cleaning robot is not much. A new simple Jacobean matrix is proposed to analyze the singularity of the robot. The singularity of the robot is obtained, which greatly simplifies the calculation of the Jacobean matrix.

Finally, the singularity analysis can effectively control the robot to avoid the singularity [9].

Therefore, it is urgent to develop an intelligent cleaning equipment for long-term self-cleaning of large condensers. Therefore, in view of the shortcomings of the current condenser cleaning technology, a new type of condenser machine design is proposed, and the application of intelligent and automatic control system to robots is not much.

Thus, in order to study the design of automatic control system for intelligent cleaning robot of condenser, the simulation model of control system model is designed by combining fuzzy algorithm with neural network, and then the model is verified, which provides a new idea for automatic control system of intelligent cleaning robot of condenser.

\section{Methodology}

\subsection{Condenser working mode}

Condenser plays the role of cooling source in the thermal cycle of steam turbine unit. Its main function is to cool the exhaust steam of steam turbine into condensate water, and establish and maintain the optimal power of steam turbine unit. The performance of condenser will directly affect the economy of the whole power plant. The main indicators to measure the performance of condenser are end difference and vacuum [10]. Steam turbine is one of the most important devices in thermal power plant. Thermal energy of steam in steam turbine is transformed into mechanical energy to provide power for generator. Condenser is an important accessory equipment of steam turbine. The exhaust steam of steam turbine is transformed into condensate water in the condenser, which reduces the volume, reduces the pressure sharply, increases the baking difference between the steams in and out of the steam turbine, and improves its performance $[11,12]$. The surface condenser structure is shown in Figure 1.

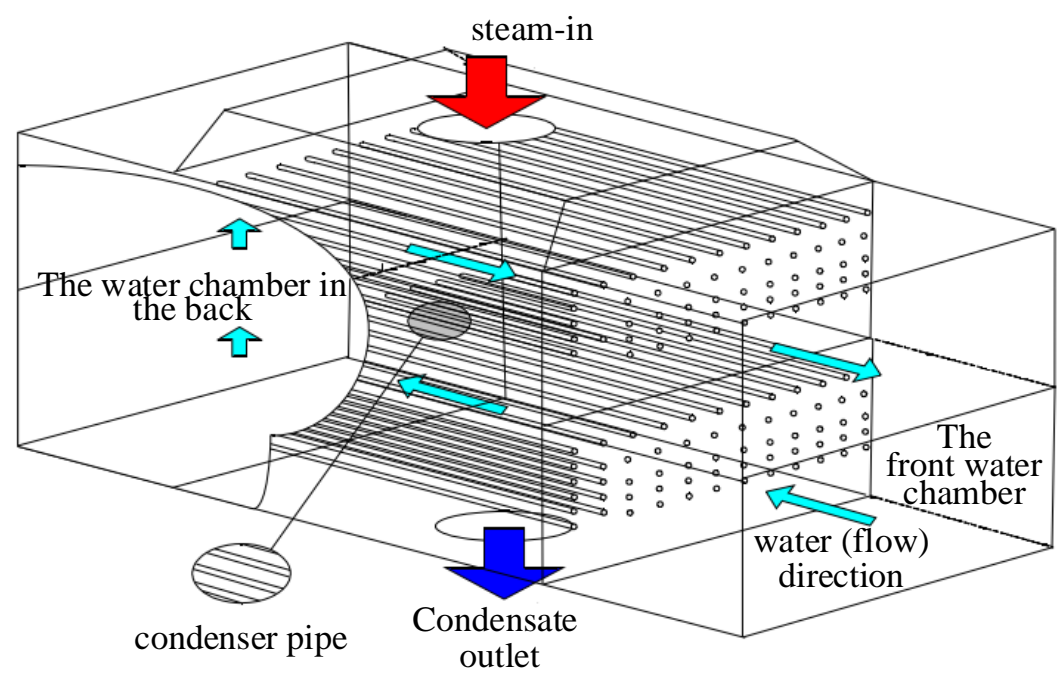

Figure 1: Structural schematic diagram of surface condenser

Most condensers use water as refrigerant.

According to the different ways of water supply, there are primary cooling water supply and secondary cooling water supply. Water supply comes from natural water sources such as rivers, lakes and seas, and the drainage is still discharged back into them, which is called primary cooling water supply or open water supply.

The water supply comes from artificial water sources such as cooling towers or cooling pools, and the drainage still returns to the cooling tower (water pool) for recycling, which is called secondary cooling water supply or closed water supply [13].
Air can be used as refrigerant in areas with special water shortage.

\subsection{Intelligent control algorithm}

At present, there is no certain definition of the term "intelligent control", which is summarized by the IEEE Control Systems Association as follows: intelligent control systems must have the ability to simulate human learning and self-adaptation [14].

As a new interdisciplinary frontier discipline, it is a new stage in the development of automatic control discipline. 
At present, the research and application of intelligent control has penetrated into aerospace, military, household appliances, service industries and other industries.

Conventional intelligent control methods mainly include fuzzy logic control, hierarchical intelligent control, neural network control, expert control and anthropomorphic intelligent control, among which the most widely used are fuzzy logic control and neural network control [15]. The main classification is shown in Figure 2.

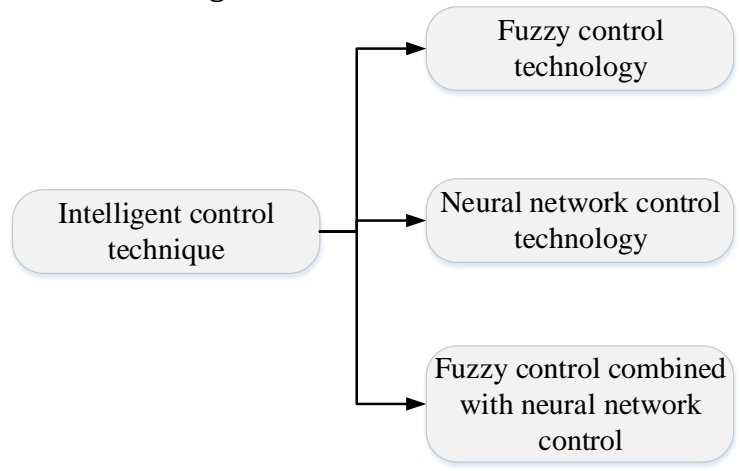

Figure 2: Structural schematic diagram of surface condenser

The application of fuzzy logic in the field of control is called fuzzy control, which is based on fuzzy mathematics. Fuzzy mathematics is the mathematics of studying fuzzy phenomena, and the concept itself is fuzzy, that is, an object cannot accurately determine whether it belongs to a concept.

The uncertainty caused by the ambiguity in the extension of the concept is called fuzziness [16] (Figure 3).

Neural network technology refers to the technology using engineering technology to simulate the structure and function of human brain neural network. Neural network is a large-scale parallel non-linear system that uses the bionics viewpoint and method to study the information processing in human brain and intelligent system [17].

Neural network is composed of information processing units (i.e. neurons) as nodes and a network topology.

Combining the fuzzy system with the neural network, the fuzzy neural network makes the fuzzy control adaptive on the one hand, and the neural network has the reasoning ability of the fuzzy control on the other.

Therefore, the fuzzy neural network has not only the ability of realizing fuzzy reasoning, but also the ability of data processing, self-adjustment, selfadaptation, self-learning and non-linear mapping [18].

It can be applied to household appliances, temperature control, image processing, pattern recognition and other fields.

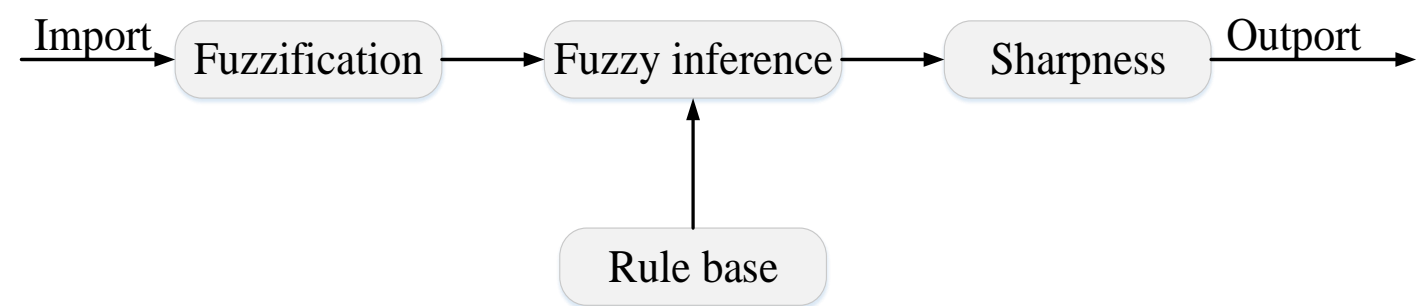

Figure 3: Basic functional structure of fuzzy controller

\subsection{Automatic control design of intelligent robot for condenser}

Control system design. In order to realize the autonomous planning and execution of the intelligent cleaning robot according to the given tasks, and to accomplish the automatic cleaning task efficiently, it is inseparable from the coordination work of each part of the robot system. The control system of intelligent cleaning robot is the core component to control the cleaning robot to complete the cleaning task. It controls all parts of the robot to coordinate the work to complete the cleaning task. Intelligent cleaning robot control system must have stable and comprehensive features. According to different functional modules, it can be divided into mobile platform control module, cleaning arm control module, high-pressure water control module, perception module, human-computer interaction control interface and communication control module, as shown in Figure 4. According to the different objects and control targets, the control system of intelligent cleaning robot can be divided into monitoring system and airborne system.

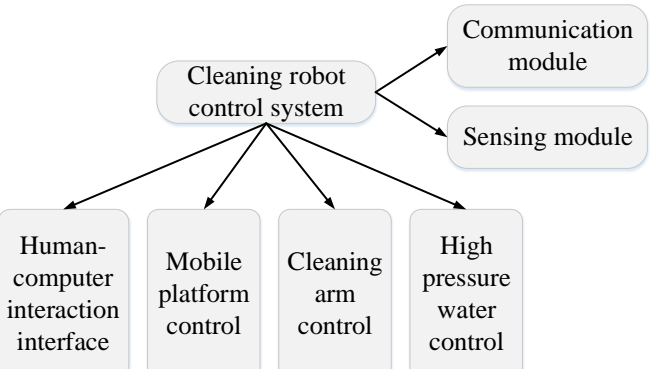

Figure 3: Control system design structural diagram

Function model of fuzzy neural system. For the fuzzy neural system, its fuzzy rules can be expressed as multiple groups of "if...then" rule statement. Assuming that the fuzzy relation synthesis method is 
used as the reasoning algorithm of the fuzzy system, the synthesis degree of the $\mathrm{i}$-th rule is as follows:

$$
\begin{aligned}
& \mu_{i}\left(X_{i}\right)=\mu_{i 1}\left(X_{i 1}\right) \mu_{i 2}\left(X_{i 2}\right) \cdots \mu_{i m}\left(X_{i m}\right)= \\
& \prod_{j=1}^{m} \mu_{i j}\left(X_{i j}\right)
\end{aligned}
$$

The total output of the fuzzy neural system is weighted by the rules. The output of the system is as follows:

$$
y=\sum_{i=1}^{n} \omega_{i} \mu_{i} / \sum_{i=1}^{n} \mu_{i}
$$

In Formula (2), $\omega_{i}$ refers to the weighting of each synthesis degree to the output.

Each subnet uses two inputs and one output. The two inputs are joint angle error and error rate, and the output is pulse value.

\subsection{Statistical analysis}

SPSS20.0 is used to establish the database, and double-entry method is used to input data to control the quality of data input. All measurement data are expressed by mean $\pm S D$, and chi-square test is used to count data.

Then normal analysis and correlation analysis are carried out on the relevant data.

\section{Results and Discussion}

\subsection{Error analysis of fuzzy neural network training}

As shown in Figure 4, the error curve obtained by taking the rotation angle of the rotating base as $0^{\circ}$ and the arm is vertical and the other joint level as the initial position of the cleaning arm can be analyzed.

It can be concluded that the output error of the fuzzy neural network is lower than the given error of 0.01 during the training process to about 85 steps, so the fuzzy neural network can get the correct and stable output.

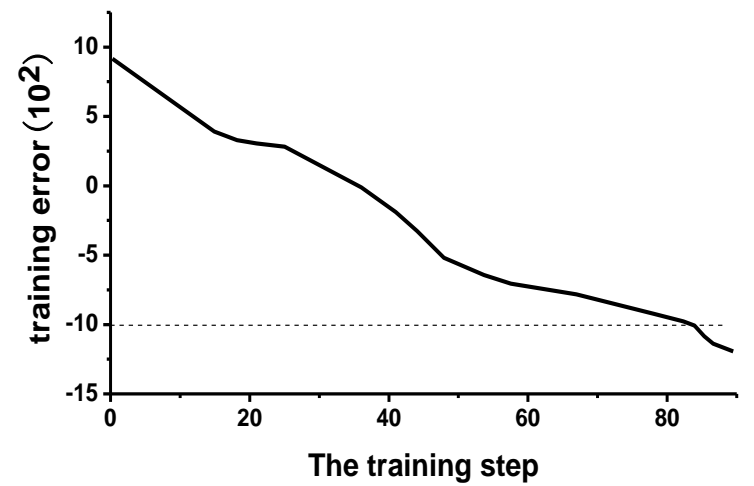

Figure 4: Training error curve of fuzzy neural network

\subsection{Step response analysis of control system}

As shown in Figure 5, through the simulation of the control system model, it can be concluded that the system has fast response speed (stable state has been reached when the time is 1.6s), high adjustment accuracy, good steady-state performance and strong robustness.

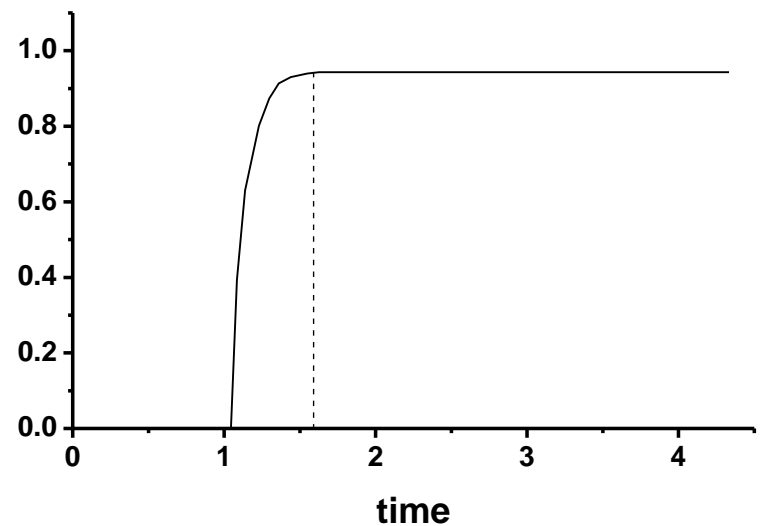

Figure 5: Training error curve of fuzzy neural network

\subsection{Step response analysis of control system}

As shown in Figure 6, the specific parameters of cleaning spray gun are set as follows: $\mathrm{L} 1=2.5 \mathrm{~m}, \mathrm{~L} 2=$ $3.0 \mathrm{~m}, \mathrm{~m} 1=40 \mathrm{~kg}, \mathrm{~m} 3=50 \mathrm{~kg}$.

The initial condition is: $\theta_{1}(0)=0 \mathrm{red}, \theta_{2}(0)=1 \mathrm{red}$, and $\hat{\theta}_{1}(0)=\hat{\theta}_{2}(0)=0 \mathrm{red} / \mathrm{s}$.

The desired trajectory is $\theta_{1}^{d}(t)=\sin (2 \pi t)$ and $\theta_{2}^{d}(t)=\cos (2 \pi t)$.

Friction term and disturbance term are $F(\dot{\Theta})=0.5 \operatorname{si} g n(\dot{\Theta})$ and

$T_{d}(\Theta, \dot{\Theta})=\left[\begin{array}{c}5 \cos (5 t) \\ 5 \sin (5 t)\end{array}\right] N \cdot \mathrm{m}$, respectively.

The quantification factor is selected as $K_{\mathrm{e} 1}=200$, $K_{e 2}=200, K_{e c 1}=1$, and $K_{e c 2}=2$; the scale factors are $\mathrm{K}_{\mathrm{u} 1}=500$ and $\mathrm{K}_{\mathrm{u} 2}=400$. From Figure $6 \mathrm{~A}$ and $\mathrm{B}$, it can be seen that the trajectory tracking curves of joint 1 and joint 2 coincide with their simulation curves and expectation curves.

From Figure 6C and D, the trajectory tracking error curves of joint 1 and joint 2 can be obtained. It is found that the initial fluctuation is large around $0.2 \mathrm{~s}$, and then the rotation frequency basically reaches a stable state.

The simulation results show that the designed fuzzy-neural controller can achieve the trajectory tracking control of cleaning spray gun, and has good dynamic and static performance and antiinterference ability. 


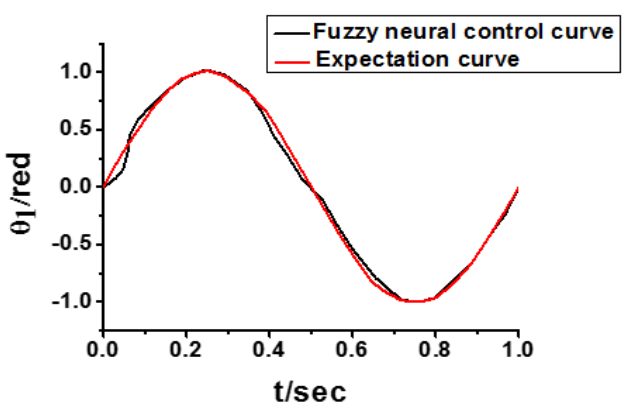

A

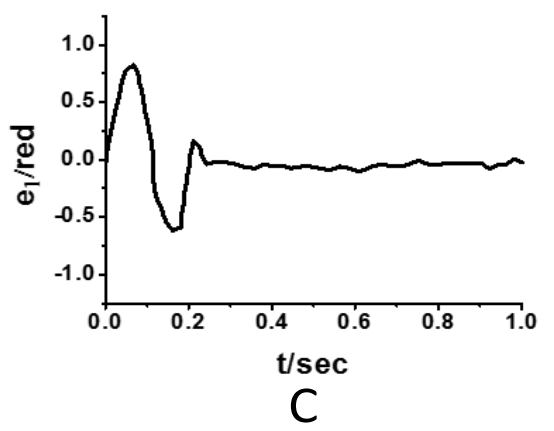

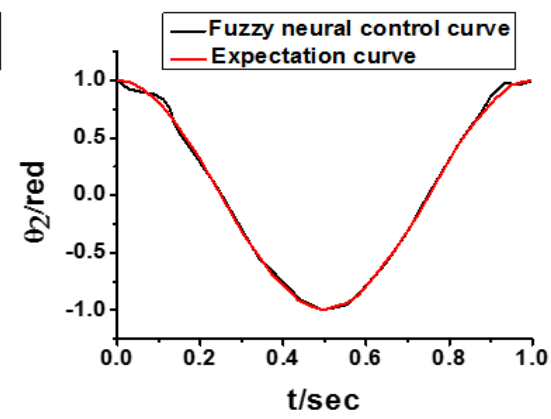

B

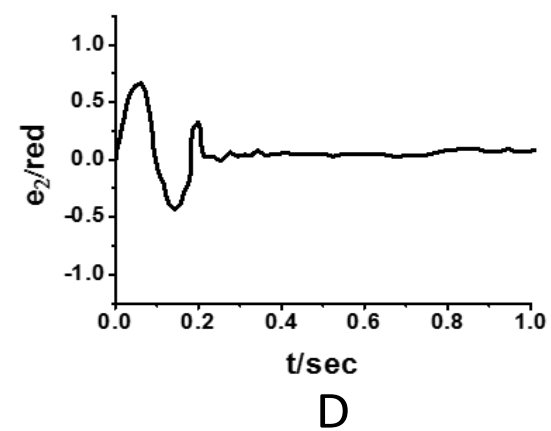

Figure 6: Joint trajectory training tracking and error curve (A, Joint 1 tracking curve; $B$, Joint 2 tracking curve; $C$, Joint 1 tracking error curve; $D$, Joint 2 tracking error curve)

\section{Conclusion}

In order to study the design of automatic control system for intelligent cleaning robot of condenser, the correlation analysis between fuzzy system and neural network system is made by understanding its working mode, and they are combined to design the simulation model of control system model, and the learning algorithm and training conditions of the controller are analyzed.

Finally, the experiment verifies that the fuzzy neural network controller can output the expected results. It suggests that the designed intelligent control system can meet the requirements of precise positioning of the cleaning target for the spray gun of the intelligent cleaning robot.

The experimental results show that the output error of the fuzzy neural network is lower than the given error of 0.01 when the training process is about 85 steps, so the fuzzy neural network can get the correct and stable output; the control system has reached a stable state when the time is $1.6 \mathrm{~s}$, and has high adjustment precision, good steady-state performance and strong robustness; and when the step response analysis of the control system is carried out, the output error of the fuzzy neural network is lower than the given error of 0.01 .

It is found that the trajectory tracking curves of joint 1 and joint 2 coincide basically with the expected curve, the tracking error curves of joint 1 and joint 2 fluctuate greatly at the initial $0.2 \mathrm{~s}$, and then the rotation frequency basically reaches a stable state.
Therefore, the fuzzy-neural controller designed here can achieve better trajectory tracking control of cleaning spray gun, and has better dynamic and static performance and anti-interference ability.

In conclusion, the design of automatic control system for intelligent cleaning robot of condenser is completed by combining the fuzzy algorithm with the neural network, and the expected results can be output through the experimental simulation, which proves that the designed intelligent control system can meet the requirements of precise positioning of automatic control of intelligent cleaning robot.

However, there are still some shortcomings in the experiment process.

For example, the deeper working mechanism of the cleaning machine is not involved.

In the future, the research will continue to deepen the working mechanism, so as to make the automatic control system of the intelligent cleaning robot of condenser more humane and intelligent.

\section{References}

[1] Luo D., Dong B., Qin W. (2017) Design and Control Methods of a Condenser Cleaning Robot. Chemical Engineering Transactions, 62, 703-708.

[2] Fucai Y., Kaihui L., Nianzheng W, et al. (2018) Study on the Design of an Embedded on-line Cleaning Condenser Tube Robot. Journal of Mechanical Transmission, (6), 17. 
[3] Liu Y., Meghat V., Machen B. (2017) Design and prototyping of an in situ system to clean a cylinder head conveying line following an engineering systems design approach. International Journal of Design Engineering, $7(2), 106-122$.

[4] Kitto J. B., Hiner L. A. (2017) Clean Power from Burning Trash. Mechanical Engineering Magazine Select Articles, 139(02), 32-37.

[5] He B., Xu S., Wang Z. (2018) A novel stiffness model for a wall-climbing hexapod robot based on nonlinear variable stiffness. Advances in Mechanical Engineering, 10(1), 1687814017752485.

[6] Reuter H. C. R., Owen M., Goodenough J. L. (2018) The Antifouling Effects of Copper-Oxide Filler Incorporated Into Paint-Based Protective Films Applied to Steam Surface Condenser Tubes. Journal of Thermal Science and Engineering Applications, 10(4), 041015.

[7] Ta V. P., Dang X. K. (2018) An Innovative Recurrent Cerebellar Model Articulation Controller For Piezo-Driven Micromotion Stage. International Journal of Innovative Computing, Information and Control, 14(4), 1527-1535.

[8] Meiru W., Yebin C. Analysis of the technology of crude oil tank cleaning. Modern Chemical Research, (1), 7.

[9] He B., Xu S., Zhou Y, et al. (2018) Mobility properties analyses of a wall climbing hexapod robot. Journal of Mechanical Science and Technology, 32(3), 1333-1344.

[10] Eyu J. I., Zhongqiao G. E. (2016) Application of A New Type Condenser Online Cleaning Device in A 600 MW Power Unit. Cleaning World, (1), 11.

[11] Samiuddin J., Badkoubeh B., Sadeghassadi M, et al. (2018) A new robust weight update for cerebellar model articulation controller adaptive control with application to transcritical organic rankine cycles. Transactions of the Institute of Measurement and Control, 0142331218807740.

[12] Steiner S., Wolf J., Glatzel S, et al. (2019) Organic synthesis in a modular robotic system driven by a chemical programming language. Science, 363(6423), eaav2211.

[13] Rani M., Kumar N., Singh H. P. (2018) Efficient position/force control of constrained mobile manipulators. International Journal of Dynamics and Control, 6(4), 1629-1638.

[14] ZHU B., LI C., WANG X, et al. (2017) Cellular decomposition algorithm of coverage path planning based on the grid map for the mobile robot. Journal of Shandong University of Technology (Natural Science Edition), (4), 4.

[15] Song W., Wang W., Guo X, et al. (2018) Autonomous Return Path Planning Method for Small Underwater Robots. Journal of Coastal Research, 83(sp1), 184-187.

[16] Khan A., Noreen I., Habib Z. On Complete Coverage Path Planning Algorithms for Nonholonomic Mobile Robots: Survey and Challenges. J. Inf. Sci. Eng. 33(1), 101-121.

[17] Juang C. F., Jhan Y. H., Chen Y. M, et al. (2018) Evolutionary Wall-Following Hexapod Robot Using Advanced Multiobjective Continuous Ant Colony Optimized Fuzzy Controller. IEEE Transactions on Cognitive and Developmental Systems, 10(3), 585-594.

[18] Cho Y. H., Lee S. C., Hong S. K. (2016) Augmentation of enhanced HDR utilizing encoder to estimate yaw-angle of cleaning robots. International Journal of Advanced Robotic Systems, 13(2), 47. 